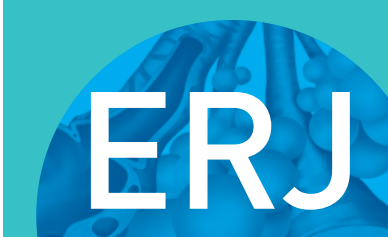

open research

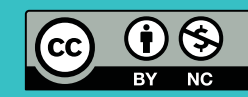

\section{Treating nosocomial pneumonia: what's new}

\author{
Frantzeska Frantzeskaki and Stylianos E. Orfanos
}

2nd Dept of Critical Care, National and Kapodistrian University of Athens and Attikon Hospital, Athens, Greece.

Correspondence: Stylianos E. Orfanos, 2nd Dept of Critical Care, Attikon Hospital, 1, Rimini St, 12462 Haidari, Greece. E-mail: stylianosorfanosuoadgmail.com

@ERSpublications

A detailed and comprehensive summary of the ERS/ESICM/ESCMID/ALAT guidelines for the management of hospital-acquired pneumonia and ventilator-associated pneumonia, published in ERJ Open Research, will assist clinicians in their tasks http://ow.ly/XC7N30k8Jhu

Cite this article as: Frantzeskaki F, Orfanos SE. Treating nosocomial pneumonia: what's new. ERJ Open Res 2018; 4: 00058-2018 [https://doi.org/10.1183/23120541.00058-2018].

Nosocomial pneumonia is an infection of lung parenchyma that occurs in patients hospitalised for more than $48 \mathrm{~h}$ after admission [1]. Hospital-acquired pneumonia (HAP) is nosocomial pneumonia in patients who do not require mechanical ventilation; while ventilator-associated pneumonia (VAP) is defined as a pneumonia developing in patients under mechanical ventilation for at least $48 \mathrm{~h} \mathrm{[2].} \mathrm{HAP} \mathrm{is} \mathrm{the} \mathrm{second}$ most common nosocomial infection, and is the most common hospital infection leading to death in critically ill patients [1]. VAP is the most frequent hospital-acquired infection in intensive care units. Depending on the diagnostic criteria used, its incidence ranges from $5 \%$ to $67 \%$ [3]. The risk of acquiring VAP is $3 \%$ per day during the first 5 days on mechanical ventilation, and it is decreased to $1 \%$ per day for the following days. HAP or VAP developing within 4 days of admission are defined as early HAP/VAP, and are usually caused by microorganisms sensitive to antibiotics. HAP or VAP occurring after 5 days of admission are defined as late-onset pneumonias, and are most commonly associated with multidrug-resistant (MDR) pathogens $[4,5]$. The mortality of late-onset VAP is higher than the respective mortality for early-onset VAP [6]. The crude mortality of nosocomial pneumonia is estimated to reach $70 \%$. Attributable mortality, which is defined as the percentage of deaths that would have been prevented in the absence of infection, is $10 \%$ [7].

Since 2005, when the American Thoracic Society/Infectious Diseases Society of America published evidence-based guidelines for HAP/VAP treatment, important progress has occurred on the elucidation of the pathophysiology of pneumonia [1]. Moreover, new studies have been published concerning the emergency of MDR pathogens and the respective treatment options. Recently, the European Respiratory Society, the European Society of Intensive Care Medicine, the European Society of Clinical Microbiology and Infectious Diseases, and the Latin America Chest Association published new guidelines for management of HAP/VAP, anticipating the European perspective on diagnosis and treatment of nosocomial pneumonia [4].

In this issue of ERJ Open Research, TORREs et al. [8] provide a summary of the International Clinical Guidelines for the management of HAP/VAP. The main topics addressed in the summary are the collection of samples of respiratory secretions, the treatment of nosocomial pneumonia according to the assumed causative pathogen, the possible combinations of antibiotics, the duration of treatment and the role of biomarkers in disease diagnosis and therapy. Given the importance of identifying the responsible pathogen, the guidelines recommend the qualitative or quantitative analysis of respiratory secretions. Although there is a lack of randomised trials comparing quantitative and qualitative cultures of the same specimen, the guidelines recommend the use of distal quantitative cultures to avoid excessive

Received: April 232018 | Accepted: April 292018

Copyright $\odot$ ERS 2018. This article is open access and distributed under the terms of the Creative Commons Attribution Non-Commercial Licence 4.0. 
antibiotic overuse. This is in accordance with cohort studies confirming the role of distal quantitative cultures in limiting the use of broad-spectrum antibiotics and restricting the antibiotic selection pressure [9]. Moreover, the guidelines clearly underline that culture specimens should be drawn before the initiation of antibiotic treatment, as the results might be influenced by the regimen used.

The treatment of nosocomial pneumonia proposed by the guidelines is based on the distinction between early and late nosocomial pneumonia, and on the presence of risk factors for antibiotic resistance. In most cases, late VAP is associated with the presence of MDR pathogens, which also depends on local microbiological data [10]. Low-risk patients are characterised by the absence of septic shock and of other risk factors for MDR pathogens, such as prior antimicrobial therapy, recent hospitalisation, advanced age and previous use of antibiotics. Therefore, the authors recommend that the use of narrow spectrum antibiotics should be based on the individual risks of each patient. Another critical issue is the administration of combinations of broad-spectrum antibiotics, in order to achieve synergistic activity against MDR pathogens. According to the authors, combination of antibiotics should be considered in patients with a high risk of MDR Gram-negative bacilli, or in patients with septic shock. Indeed, several studies have shown that combination therapy is associated with a lower risk of death than monotherapy [11]. However, the authors clearly outline that, depending on the antibiogram data, de-escalation of treatment may be considered. GARNACHO-MONTERO et al. [12] have shown the importance of de-escalation of empirical therapy in lowering the mortality in patients with severe sepsis and septic shock.

Regarding the duration of treatment, the authors recommend a 7-8-day course of antibiotics, in the absence of pulmonary empyema, lung abscess, cavitation and necrotising pneumonia [8]. After 3 days of treatment, re-evaluation of the patient is suggested. The role of biomarkers and of clinical assessment has been interestingly underlined in the present summary. Serial evaluations of the Clinical Pulmonary Infection Scale might help in the early recognition of patients with poor clinical response to treatment, while the Sequential Organ Failure Assessment score is associated with survival, as has been depicted in several studies $[13,14]$. Concerning biomarkers, procalcitonin (PCT) is a strong predictor of risk of death. Moreover, PCT is a promising biomarker of treatment duration, and several PCT-guided algorithms for VAP treatment have been studied [15]. However, as the authors highlight, the clinician should combine serial measurements of PCT with clinical assessment of the patient to decide on the initiation or completion of antibiotic therapy.

TORRES et al. [8] are to be congratulated for providing a detailed and comprehensive summary of the international clinical guidelines for the management of HAP/VAP; this work will strongly assist clinicians in their tasks. The guidelines are evidence-based and might be followed by all health professionals dealing with patients with HAP/VAP. Future clinical trials should define the role of new rapid diagnostic tests in $\mathrm{VAP} / \mathrm{HAP}$ diagnosis, the use of inhaled antibiotics in VAP/HAP therapy and the contribution of biomarkers in guiding clinical decisions.

Conflict of interest: None declared.

\section{References}

1 Guidelines for the management of adults with hospital-acquired, ventilator-associated, and healthcare-associated pneumonia. Am J Respir Crit Care Med 2005; 171: 388-416.

Chastre J, Fagon JY. Ventilator-associated pneumonia. Am J Respir Crit Care Med 2002; 165: 867-903.

Timsit JF, Esaied W, Neuville M, et al. Update on ventilator-associated pneumonia. F1000Research 2017; 6: 2061.

Torres A, Niederman MS, Chastre J, et al. International ERS/ESICM/ESCMID/ALAT guidelines for the management of hospital-acquired pneumonia and ventilator-associated pneumonia: Guidelines for the management of hospital-acquired pneumonia (HAP)/ventilator-associated pneumonia (VAP) of the European Respiratory Society (ERS), European Society of Intensive Care Medicine (ESICM), European Society of Clinical Microbiology and Infectious Diseases (ESCMID) and Asociacion Latinoamericana del Torax (ALAT). Eur Respir J 2017; 50: 1700582 .

5 Martin-Loeches I, Torres A, Rinaudo $\mathrm{M}$, et al. Resistance patterns and outcomes in intensive care unit (ICU)-acquired pneumonia. Validation of European Centre for Disease Prevention and Control (ECDC) and the Centers for Disease Control and Prevention (CDC) classification of multidrug resistant organisms. J Infect 2015; 70: $213-222$.

6 Nguile-Makao M, Zahar JR, Francais A, et al. Attributable mortality of ventilator-associated pneumonia: respective impact of main characteristics at ICU admission and VAP onset using conditional logistic regression and multi-state models. Intensive Care Med 2010; 36: 781-789.

7 Melsen WG, Rovers MM, Koeman M, et al. Estimating the attributable mortality of ventilator-associated pneumonia from randomized prevention studies. Crit Care Med 2011; 39: 2736-2742.

8 Torres A, Niederman MS, Chastre J, et al. Summary of the international clinical guidelines for the management of hospital-acquired and ventilator-acquired pneumonia. ERJ Open Res 2018; 4: 00028-2018.

9 Bonten MJ, Bergmans DC, Stobberingh EE, et al. Implementation of bronchoscopic techniques in the diagnosis of ventilator-associated pneumonia to reduce antibiotic use. Am J Respir Crit Care Med 1997; 156: 1820-1824. 
10 Diaz E, Martin-Loeches I, Valles J. Neumonia nosocomial [Nosocomial pneumonia]. Enferm Infecc y Microbiol Clin 2013; 31: 692-698.

11 Kumar A, Zarychanski R, Light B, et al. Early combination antibiotic therapy yields improved survival compared with monotherapy in septic shock: a propensity-matched analysis. Crit Care Med 2010; 38: 1773-1785.

12 Garnacho-Montero J, Gutiérrez-Pizarraya A, Escoresca-Ortega A, et al. De-escalation of empirical therapy is associated with lower mortality in patients with severe sepsis and septic shock. Intensive Care Med 2014; 40: $32-40$.

13 Luna CM, Blanzaco D, Niederman MS, et al. Resolution of ventilator-associated pneumonia: prospective evaluation of the clinical pulmonary infection score as an early clinical predictor of outcome. Crit Care Med 2003; 31: 676-682.

14 Seligman R, Meisner M, Lisboa TC, et al. Decreases in procalcitonin and C-reactive protein are strong predictors of survival in ventilator-associated pneumonia. Crit Care 2006; 10: R125.

15 Luyt CE, Guerin V, Combes A, et al. Procalcitonin kinetics as a prognostic marker of ventilator-associated pneumonia. Am J Respir Crit Care Med 2005; 171: 48-53. 